\title{
Pulmonary Tuberculosis Treated with “Onnetsu” Far Infrared Rays: Case Report
}

\author{
Aranda Ventura José, Tatsumura Hillyer Kazuko* \\ Director, Gaia Holistic Health, USA \\ *Corresponding author: Tatsumura Hillyer Kazuko, Director, Gaia Holistic Health, USA
}

\begin{abstract}
Every year, 220,000 new cases of tuberculosis are registered in the Americas, and more than 50,000 die as a result of this disease. It is worth noting that tuberculosis is both preventable and curable, huevero it still is one of the most important transmitted infections in the world. Multiple factors explain this situation, one of them being the lack of commitment to treatment, due the amount of pills the patient must take and the side effects they may have. This raises the necessity of developing an efficient therapy that decreases negative side effects and permit proper adherence to the treatment. To this day there is no registered use of "Onnetsu" far infrared rays on patients suffering from tuberculosis. Take the case of a 67 year-old woman diagnosed with pulmonary tuberculosis (TB) based on clinical, radiological and bacteriological evidence, for which she was admitted in the National Health Strategy for the Prevention and Control of Tuberculosis (ESNPCT) and was receiving pharmacological treatment for 1 month (Rifampicin 300mg,Ethambutol 400mg, Isoniazid 100mg, Pyrazinamide 500mg), which she abandoned when she began having adverse reactions (nausea, vomiting, lose of appetite, weakness and dyspepsia). Due to this situation she went to the Traditional Medical Institute-IMET ESSALUD, where the diagnosis of pulmonary tuberculosis was confirmed and then signed and informed consent form where she agreed to receive "Onnetsu" far infrared ray treatment, which were applied to the skin, focussing on the thorax, done in one hour sessions, 3 times per week for 18 weeks, during which she received no pharmacological treatment. At the end of the treatment, betterment in both the clinical and radiological pictures and an adequate bacteriological response resulted in her being discharged from ESNPCT. Monitoring was done over the next 6 years y no evidence of active TB was found. This report could be the first step in opening the studies on the use of far infrared rays as a potential treatment for pulmonary tuberculosis.
\end{abstract}

\section{Introduction}

Tuberculosis is caused by Mycobacterium tuberculosis, a bacterium that frequently affects the lungs. This disease is curable and preventable. Tuberculosis is transmitted from person to person through the air. It is calculated that around a third of human population has latent tuberculosis and as result have a small risk, around $10 \%$, of being infected with $\mathrm{TB}$, this risk increases in people with weakened immune systems, such as people suffering from HIV/AIDS, malnutrition or diabetes as well as those who consume tabaco. Over the course of a year, a patient with tuberculosis can infect from 10 to 15 people through close contact; if patients are not given proper treatment, over two thirds of them die [1]. Tuberculosis is the ninth leading cause of death worldwide and first among infectious diseases, over HIV/AIDS. In 2016 the estimated death toll via TB was 1.3 million in HIV-negative people and 374,000 HIV-positive people. The estimated number of people who caught the disease in that same year was 10.4 million: $90 \%$ of them adults, 65\% male and 10\% HIV-positive. Drug resistant tuberculosis is still a major threat; in 2016 there were 600,000 new cases of Rifampicin resistance, the most efficient antibiotic; 490,000 of these cases were multiresistant. $47 \%$ of cases occurred in China, India and Russia [2].

Over the last couple of years in Peru, cases of pan sensitive tuberculosis have decreased, and cases of multidrug resistant tuberculosis (TB-MDR) have increased, except from 2009 to 2011. Around $90 \%$ of extensively drug-resistant tuberculosis (XDR-TB) cases where reported in Lima and Callao. Pan sensitive TB mostly 
affects the 20-59 demographic and occurs mostly in departments with in jungles (Loreto, Ucayali, Madre de Dios) and Lima-Callao) [3]. The basis for tuberculosis treatment is the Directly Observed Therapy Short-Course (DOTS). Despite the fact that Peru was a model for its implementation, TB is still on the rise, and an even more dangerous situation is developing due to the increasing cases of TB-MDR. Some of the reasons for this are the delay in diagnosis and treatment; the lack of commitment to the treatments; the small number of facilities available and the mismanagement of adverse effects resulting from treatment [3]. Research and development of new pharmacological treatments and vaccines is ongoing, but slow. It is for these reasons that the investigation in new, nonconventional therapies with promising efficiency are justified, with few side effects and permitting a proper adherence to the treatment. There is increasing evidence that far infrared rays (FIR), a type of non-invasive physiotherapy, improves the well-being of patients suffering from cardiovascular disease, diabetes, chronic kidney disease, according to a systematic revision, where it was concluded that its action mechanism could be directly related to an increase in nitric oxide synthases, as well as regulating some circulating microRNAs; this can be completely beneficial for the treatment of certain chronical diseases, due to no adverse effects being reported [4]. In regards to infectious diseases, a satisfactory response was reported after using FIR on 5 patients with Sporotrichosis, a skin condition [5], there are no other reports of FIR use on other types of infections. Due to the fact that in the pathogenesis of tuberculosis, nitric oxide is a key and effective molecule in the fight against the Mycobacterium tuberculosis, the potential of nitric oxide based treatments is being investigated [6], in this context it's valid to pose that the use of FIR could be useful in the treatment of infections such as pulmonary tuberculosis.

\section{Case Report}

67 year-old women, with a clinical history of Parkinson's disease (under treatment with 25/250 mg Carbidopa/Levodopa 3 time per day) and arterial hypertension (under treatment with Enalapril $20 \mathrm{mg} 1$ time per day), diagnosed with pulmonary tuberculosis 0n 21/10/11 (based on the clinical evaluation, thorax radiography done on 14/10/11 and a ++ sputum smear microscopy, on two samples collected on the 19 and 21 of October 2011) in the Morona Cocha Health Centre, where she received treatment from scheme 1, the first phase ( which was done partially over a month from 21/10/11 to 21/11/11); Rifampicin $300 \mathrm{mg}$, Ethambutol 400 $\mathrm{mg}$, Isoniazid $100 \mathrm{mg}$, Pyrazinamide $500 \mathrm{mg}$. the patient had to take, every day, a total of 13 pills, 3 for Parkinson's, 1 for arterial hypertension and 9 for pulmonary tuberculosis. She abandoned treatment after presenting adverse effects such as vomiting, loss of appetite and weakness. The patient was clinically evaluated on 24/11/11, at the Institute of Traditional Medicine -IMET of the Social Secure of Health-EsSalud, Iquitos- Peru, presenting symptoms such as productive cough, difficulty breathing (disnea), thoracic pain, nocturnal diaphoresis, weakness and loss of weight, regarding the side effects, she stated the nausea, the vomit, and the epigastralgia decreased since she stopped doing the treatment. Biological functions were normal, except: loss of appetite, nocturnal diaphoresis, a slight feeling of thirst and insomnia. The physical exam presented: bad general condition, fever $\left(37.8^{\circ} \mathrm{C}\right)$, tachypnea (FR 23 breath/min), FC 90 beats/min), PA $140 / 80 \mathrm{~mm} / \mathrm{Hg}$, weight $41.8 \mathrm{~kg}$, height $1.47 \mathrm{~cm}$ (IMC 19.4); when she was auscultated she presents rattles in the superior lobe of the right lung, when she coughed. On the $26 / 11 / 11$, the thoracic scan shows: some abnormal opacity in the superior lobe of the right lung, including oxidative infiltrations, compatible with a specific process in progress. The hematology and the biochemistry at this time were normal. On 30/11/11, the direct examination of septum smears for acid-fast bacilli reports: BAAR $2(+)$.

We present to the patient the possibility of an alternative treatment, including risks and benefits of it, she signed the informed consent papers and she decided to follow the treatment with no invasive technology, commonly used in physiotherapy that emits far infrared rays "ONNETSU", which is between 69 to $70{ }^{\circ} \mathrm{C}$, which she could see on the device, with wave length range of 8 to $10 \mathrm{um}$, the treatment plan consisted in applying this technology on a cotton sheet that covers the body of the patient, with special emphasis on the back and front of thorax, one hour sessions were carried out three times a week during 18 weeks. The sessions were started on 1/12/11 at the Institute of Traditional Medicine -IMET de ESSALUD, the surgeon of the Institute, was responsible of applying the therapy and doing the patient's monitoring. Nine days after the treatment started with FIR "ONNETSU", the patient stated feeling better. The cough, moderately productive, the dyspnea, nocturnal diaphoresis, the weakness mostly decreased; the thoracic pain was gone, appetite and sleeping improved; the thirst decreased. Decreases in nausea, vomiting and epigastralgia were reported as well. The physical exam showed a patient in regular general condition, without fever, still with taquipnea (FR 19) but in decreased state, without changes when she was auscultated in her right lung. The direct examination of sputum smears for acidfast bacilli reports: BAAR. Thirty days after the treatment started the patient stated feeling much better and recovering, cough still was moderately productive, mild dyspnea, remission of nocturnal diaphoresis. Normal biological Functions. In the physical exam, showed a patient improving her general condition, without fever, with mild taquipnea (FR 17), when she was auscultated we heard well through the vesicular murmuring in both lungs. The direct examination of sputum smears for acid-fast bacilli reports (30712/11) was negative.

Since the treatment with FIR ONNETSU started (1/12/11) the first monitoring was done during the next four and a half months $(12 / 4 / 12)$, at the IMET-ESSALUD and at the laboratory of the "Medical Institute Amazon of Science", at the same time the patient attend to her monitoring at MINSA (Centro de Salud Morona Cocha). The clinical controls during this time showed the patient with 
increasing improvements, with the direct examination of sputum smears for acid-fast bacilli reports was negative on the following dates: 10/01/12, 13/02/12, 10/03/12 and 09/04/12 (see graphic 1). On $10 / 4 / 12$ she was evaluated by a nurse incentro de Salud Morona Cocha-MINSA and on 12/4/12, she was evaluated by a doctor of "Estrategia Sanitaria Nacional de Prevencióny Control de la Tuberculosis", of the same medical centre, and then he recorded in the patient's medical record "TBC WAS CURED" and then she was discharged.

In spite of the patient showing no symptoms, her monitoring was continued; her direct examination of sputum smears for acidfast bacilli report was negative. (15/11/12). Radiological scans shows the following improvements: The report on 23/3/12 shows a dramatical reduction of the exudatives agglomerations in the upper right middle lobe, observing better ventilation, indicating improvement in comparison with the scan done on $26 / 11 / 11$ (basal); the scan done on 15/11/12 in comparison with the scan done on $23 / 3 / 12$, shows improvement, decrease in size and quantity of the exudations described. We also held a control on $2 / 9 / 13$, the patient continued to be without symptoms and her direct examination of sputum smears for acid-fast bacilli report was negative. From 2014 to 2017 some home visits were done, showing that the patient had no symptoms of a respiratory disease. On May 2018, she presented productive cough and she was evaluated by a pulmonologist, doctor in Chief of "Estarategia Sanitaria Nacional de Prevencion y Control de Tuberculosis" of the Health Center of Morona Cocha-MINSA, after personally evaluating her and in accordance with the thoracic scan done (15/5/18, in which reports the presence of fibro topic images of a residual aspect in the upper right middle lobe), and the last direct examination of sputum smears for acid-fast bacilli $(21 / 5 / 18)$, certified that there is no pulmonary tuberculosis and he only prescribed: Acetylcysteine 1 tablet/day for a month. See timeline (Figure 1) (Table 1).

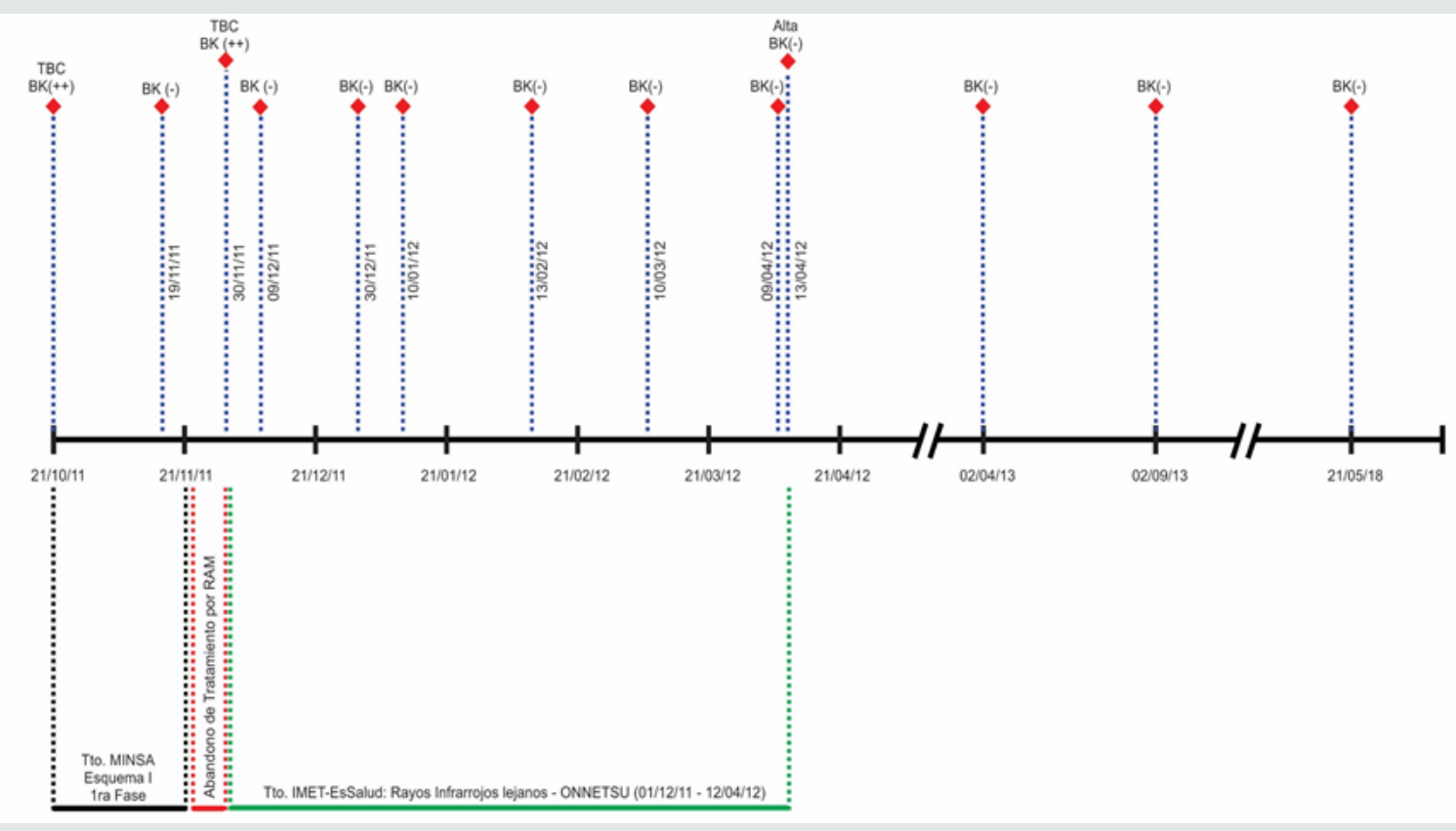

Figure 1: Timeline of the evolution of the patient with Pulmonary Tuberculosis treated with FIR- ONNETSU.

Table 1: Results of the Evaluation of direct examination of sputum smears for acid-fast bacilli of the patient with Pulmonary Tuberculosis treated with FIR-ONNETSU.

\begin{tabular}{|c|c|c|c|}
\hline \multicolumn{2}{|c|}{ Laboratory of Centro de Salud Morona Cocha -MINSA } & \multicolumn{2}{|c|}{ Laboratory of Instituto Medico Amazónico de Ciencias } \\
\hline Dates & Results of BK & Dates & \\
\hline $19 / 10 / 11$ & ++ & & \\
\hline $21 / 10 / 11$ & ++ & & $+5 / 11 / 11$ \\
\hline $19 / 11 / 11$ & - & $9 / 12 / 2011$ & + \\
\hline & - & & \\
\hline $30 / 12 / 11$ & - & & \\
\hline $10 / 1 / 2012$ & - & & \\
\hline $13 / 02 / 12$ & - & & \\
\hline
\end{tabular}




\begin{tabular}{|c|c|c|c|}
\hline $10 / 3 / 2012$ & - & & \\
\hline $9 / 4 / 2012$ & - & & \\
\hline $13 / 04 / 12$ & - & & \\
\hline $2 / 4 / 2013$ & - & & \\
\hline $2 / 9 / 2013$ & - & & \\
\hline & & & $21 / 05 / 18$ \\
\hline
\end{tabular}

\section{Discussion}

The clinical, bacteriological and radiological remission of the pulmonary tuberculosis using only FIR hasn't been reported until today. Although, it should be noted seeing the scans and their respective reports, a slow resolution of the radiological abnormalities is evidenced, which matches with other studies [7], so regarding the scientific literature, the thoracic scan is not the best method to monitoring the response of the treatment. Instead, a clinical evaluation and bacteriologic monitoring of the treatment must be done in order to be able to define if the patient has responded properly [7]. In the Infectiology field, we have only one report of a series of cases of patients with Sporotrichosis treated successfully with FIR [5]. Also, there are reports of experimental studies like: FIR healed the wounds significantly more quick in rats skin, increasing the regeneration of collagen and the infiltration of fibroblasts which express the growing factor TGF-BETA1 [8], the FIR has a biological effect related to nitric oxide for increasing the microcirculation of the skin in rats, which could have clinical utility for the treatment of ischemic diseases [9], the treatment with FIR during next 48 hours after produced decrease of $11.8 \%$ in the proliferation of melanoma merino cells [10]. A systemic revision, has raised that the mechanism of action of FIR could be closely connected with the increased Nitric oxide sintetasa endothelial as well as in the Nitric Oxide production and could be responsible of the modulate of the profiles of some miARNS in circulation [4], on the other hand, we know that in the pathogenesis of the tuberculosis, the molecule of Nitric Oxide is a key and effective against the Mycobacterium tuberculosis [6], on this context it is possible that the FIR have been acting in this way. There are no reports of side effects using FIR [4], since it is no invasive therapy and it is safe, at this case there is no reports of side effects at all regarding the application of this technology. This issue favoured the adherence to the Treatment Plan. We recommend doing prospective studies in order to evaluate if the effect of this Treatment Plan is replicable and if it could be useful in Pulmonary Tuberculosis Treatment.

\section{References}

1. (2016) Qué es la tuberculosis y cómo se trata? Organización Mundial de la Salud.

2. (2017) Informe mundial sobre la tuberculosis 2017-Sinopsis. Organización Mundial de la Salud.

3. Llanos-Zavalaga LF, Velásquez-Hurtado JE, García PJ, Gottuzzo E (2012) Tuberculosis y salud pública: derechos individualesy derechos colectivos? Rev Peru Med Exp Salud Pública 29(2): 259-264.

4. Shanshan Shui, Xia Wang, John Y Chiang, Lei Zheng (2015) Far-infrared therapy for cardiovascular, autoimmune, and other chronic health problems: A systematic review. Experimental Biology and Medicine 240: 1257-1265.

5. Hiruma M, Kawada A, Noguchi H, Ishibashi A, Conti Diaz IA (1992) Hyperthermic treatment of sporotrichosis: experimental use of infrared and far infrared rays. Mycoses 35(11-12): 293-299.

6. Jamaati H, Mortaz E, Pajouhi Z, Folkerts G, Movassaghi M, et al. (2017) Nitric oxide in the pathogenesis and treatment of tuberculosis. Front Microbiol 8: 2008.

7. Miranda G, Diaz J, Arancibia P, Antolini M, Diaz C, et al. (2004) Manifestaciones radiográficas de la tuberculosis pulmonar. Rev Chil Radiol 10: 178-182.

8. Toyokawa H, Matsui Y, Uhara J, Tsuchiya H, Teshima S, et al. (2003). Promotive effects of far-infrared ray on full-thickness skin wound healing in rats. Exp Biol Med (Maywood) 228(6): 724-729.

9. Shi Yau Yu, Jen Hwey Chiu, Shiaw Der Yang, Yu Chen Hsu, Wing Yiu Lui, et al. (2006) Biological effect of far-infrared therapy on increasing skin microcirculation in rats. Photodermatol Photoimmunol Photomed 22(6): 78-86.

10. Ting Kai Leung, Chin Feng Chan, Ping Shan Lai, Chih Hui Yang, Chia Yen Hsu, et al. (2012) Inhibitory effects of far-infrared irradiation generated by ceramic material on murien melanoma cell growth. International Journal of Photoenergy p. 8.

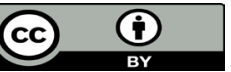

This work is licensed under Creative Commons Attribution 4.0 License

To Submit Your Article Click Here: Submit Article

DOI: 10.32474/OAJCAM.2019.01.000123

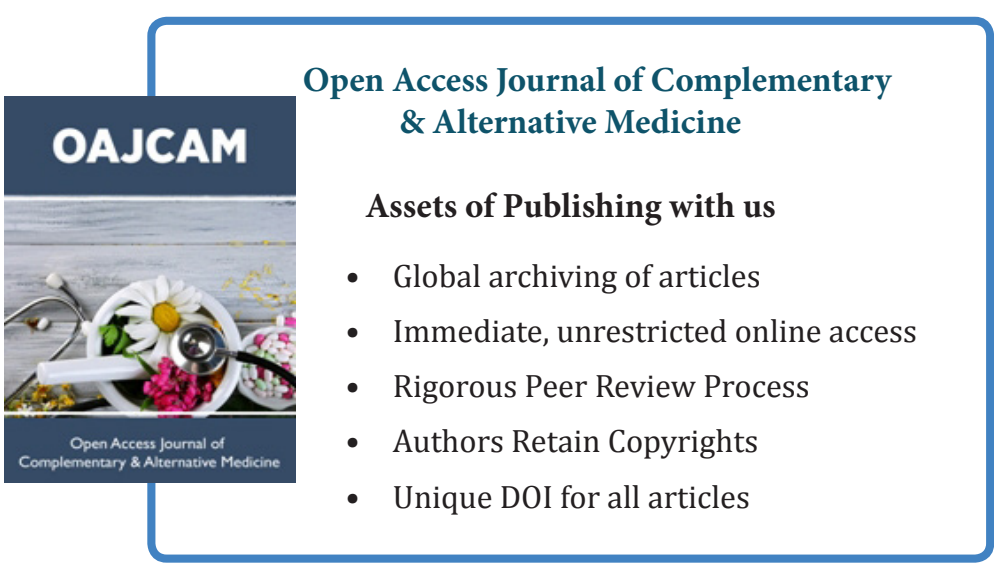

